\title{
RHEOLOGICAL PROPERTIES OF HUMAN SALIVA
}

\author{
H. A. Waterman, ${ }^{1}$ C. Blom, ${ }^{1}$ H. J. Holterman, ${ }^{1}$ E. J. 's-Gravenmade ${ }^{2}$ \\ and J. MELlema' \\ 'Rheology Group, Department of Applied Physics, Twente University, P.O. Box 217, Enschede and \\ ${ }^{2}$ Laboratory for: Materia Technica, University of Groningen, P.O. Box 72, Groningen, The Netherlands
}

\author{
(Accepted 28 January 1988)
}

\begin{abstract}
Summary-From measurements with a Couette-type viscometer provided with a guard ring it was shown that at the saliva-air interface a protein layer is adsorbed. Measurements of the surface shear modulus of this layer on saliva of 7 healthy subjects were performed at a frequency of about $70 \mathrm{~Hz}$ and a temperature of $25^{\circ} \mathrm{C}$. For a surface age of about $1.5 \mathrm{~h}$ the surface shear modulus and the surface viscosity were in the order of $1 \mathrm{Nm}^{-1}$ and $10^{-3} \mathrm{Nm}^{-1} \mathrm{~s}$, respectively. From ellipsometric measurements it was found that the thickness of the protein layer was approx. $100 \mathrm{~nm}$ and, using this value, it could be concluded that the shear modulus and the dynamic viscosity were in the order of $10^{7} \mathrm{~Pa}$ and $10^{4} \mathrm{~Pa}$ s, respectively. The layer appeared to be fragile. Even shear deformation amplitudes of $4 \times 10^{-5}$ are too high to assure linearity. The complex viscosity $\left(\eta=\eta^{\prime}-i \eta^{\prime \prime}\right)$ of the bulk liquid of human submandibular saliva below the absorbed layer was measured in the frequency range $70 \mathrm{~Hz}-200 \mathrm{kHz}$ with 3 torsional resonators, each for a different frequency, and a Ni-tube resonator. It was concluded, that the real part of the complex viscosity $\left(\eta^{\prime}\right)$ decreases from $1.1 \mathrm{mPa} s$ at $70 \mathrm{~Hz}$ to a value of $0.95 \mathrm{mPa} \mathrm{s}$ at high frequencies. Except at the lowest frequency $(70 \mathrm{~Hz})$, the value of $\eta^{\prime \prime}$ was too small to be detected.
\end{abstract}

\section{INTRODUCTION}

Saliva serves diverse functions in the human mouth. Among these, lubrication is very important, facilitating the movements of the tongue and the lips during chewing and swallowing and being essential for clearly articulated speech. The efficacy of saliva as a lubricant will depend on its viscosity and how this changes with shear rate. The shear rate can obtain high values, e.g. 160 and $60 \mathrm{~s}^{-1}$, during speaking and swallowing, respectively (Balmer and Hirsch, 1978). We conclude that the rheological properties of saliva are of major importance in these processes. Until the 1970 s most rheological measurements of saliva were limitcd almost cxclusively to Ostwald type U-tube viscometers or their modifications. A more sophisticated experimental technique was used by Davis (1971): with a Weissenberg rhcogoniometer he mcasured the real and imaginary parts, $G^{\prime}$ and $G^{\prime \prime}$, respectively, of the shear modulus of saliva in the frequency range $2.5 \times 10^{-3}-10 \mathrm{~Hz}$. At the lowest frequency the dynamic viscosity, $\eta^{\prime},\left(G^{\prime \prime}=\omega \eta^{\prime}\right)$ attained values of about $40 \mathrm{~Pa}$ s. The values of $G^{\prime}$ and $G$ " as a function of the frequency are similar to those found for sputum.

Waterman, 's-Gravenmade and van der WallenMijnlieff (1985) found that at the saliva-air interface a mechanically rigid layer is adsorbed. We have now measured the surface shear modulus of this layer, and the complex viscosity of the bulk liquid below the surface layer between $70 \mathrm{~Hz}$ and $200 \mathrm{kHz}$.

\section{MATERIALS AND METHODS}

\section{Salivas}

Saliva was collected from 7 healthy males (ages 23-48 yr) between 09.00 and $11.00 \mathrm{~h}$. In collection of unstimulated whole saliva the subjects were seated comfortably in an area with minimal external distractions, instructed to sit quietly and to minimize all movements. Stimulated submandibular and parotid saliva were collected using a Lashley cup (Lashley, 1916) and a modified Schneyer's appliance (Schneyer, 1955). Stimulation was by application of 2 per cent citric acid to the tongue with a cotton-tip applicator. Salivary flow rate was estimated by recording the approximate volume collected during a $10 \mathrm{~min}$ period. Values were within the range $300-1000 \mu \mathrm{l} / \mathrm{min}$, which is typical (Mandel and Wotman, 1976).

After a saliva sample had been collected it was studied immediately at a controlled temperature of $25^{\circ} \mathrm{C}$. Within the biological range variations the measurements showed common trends. In this account for each kind of measurement an example is selected that shows the typical characteristics of the saliva samples investigated.

\section{Apparatus}

Steady-state measurements. These were performed with a Contraves Low Shear 30 viscometer provided with a home-made guard ring. A schematic representation of the measuring unit is shown in Fig. 1. The apparatus measures the torque required to keep the bob in a fixed position when the cup is rotating with a certain angular velocity. The guard ring, which is connected to the main frame, can be moved in a vertical direction. In the upper position the guard ring does not touch the sample and the torque measured is originated from the surface layer and the bulk liquid below this layer. In the lower position the torque originated from the surface layer is eliminated, because the ring-shaped surface between the bob and the guard ring is now at rest.

Torsional resonators for measurement of surface shear modulus. These measurements were performed with a modified version of a $70 \mathrm{~Hz}$ torsional resonator, first developed in our laboratory (Blom and Mellema, 1984). A simplified diagram of the torsional resonator is given in Fig. 2. The (nearly) biconical bob is machined from non-magnetic steel and pro- 

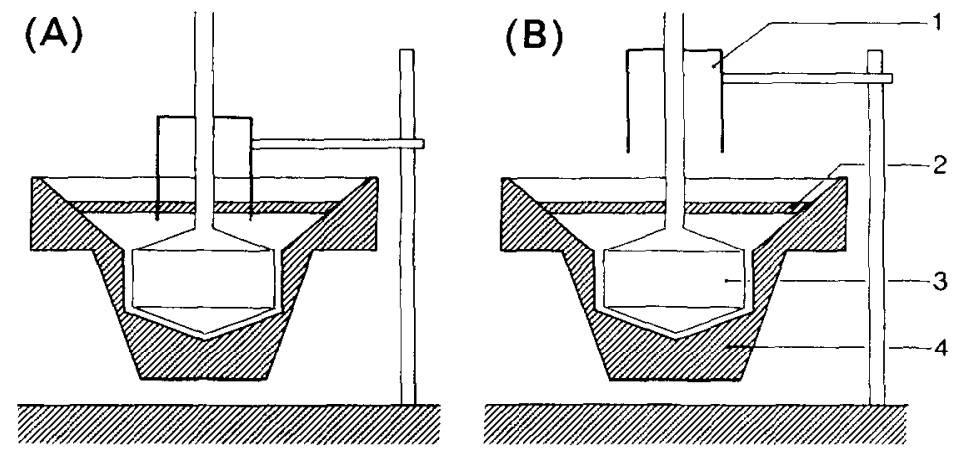

Fig. 1. Schematic representation of the measuring unit of the Contraves Low Shear 30. (A) Guard ring in the lower position. (B) Guard ring in the upper position. 1-Guard ring; 2-surface layer; 3-bob; 4-cup.

vided with a cylindrical magnet, which has been glued into it. The purpose of the Tcflon ring, inserted in the sample holder, is to ensure a flat surface during surface shear measurements. In contrast with the original apparatus the momentum of inertia of this bob is made as small as possible to enhance the sensitivity of the apparatus for surface shear measurements.

Torsional vibrations are excited and detected with coils positioned just outside the sample holder. The output of the detecting coil is proportional with $\theta, \theta$ being the angular displacement of the bob. Temperature control is established by water circulated from a thermostat through the double-walled, glass sample holder. Filling and emptying of the sample holder is performed using dry nitrogen gas.

Measurements consisted of the determination of the resonance curves of the resonator under different loading conditions as described. From each curve the bandwidth $(H B$; defined as the difference between the frequencies for which $\theta$ equals $1 / 2 \sqrt{2}$ times its maximum value) and the resonance frequency $\left(f_{0}\right)$ were obtained. The sample holder of the torsional resonator could be closed, thus assuring a minimum of evaporation.

Bulk measurements with torsional resonators. Three torsional resonators were applied in the frequency

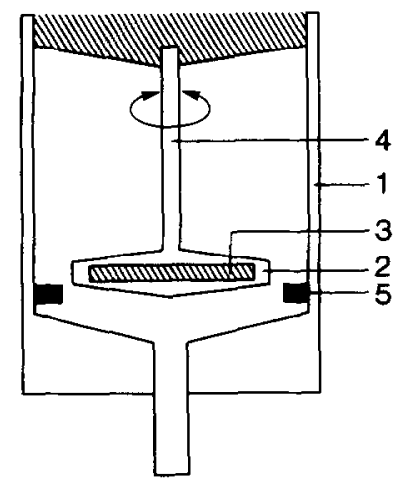

Fig. 2. Simplified diagram of the torsional resonator. 1-Sample holder, inside circular, outside square; 2-resonator mass; 3-magnet; 4-torsional spring; 5 -teflon ring. range $70 \mathrm{~Hz}-2.5 \mathrm{kHz}$. At the lowest frequency $(70 \mathrm{~Hz})$ the modified resonator was applied. At the frequencies $\sim 720 \mathrm{~Hz}$ and $\sim 2.500 \mathrm{~Hz}$ the resonators (numbers $3 \& 4$ ) of Blom and Mellema (1984) were applied because smaller samples could be used. The sample holders could be closed to avoid evaporation.

Bulk measurements with the $\mathrm{Ni}$-tube resonator. In the frequency range $3.7-200 \mathrm{kHz}$, measurements were performed with a Ni-tube resonator (Waterman et al., 1979; Oosterbroek et al., 1980). The resonator (Fig. 3) is composed of a $\mathrm{Ni}$ tube (outer dia $4 \mathrm{~mm}$, wall thickness $0.1 \mathrm{~mm}$ ), which is mounted on 4 stainless-steel wires (dia $0.1 \mathrm{~mm}$ ). The wires, provided with small knots, are led through 4 holes (dia $0.15 \mathrm{~mm}$ ) drilled in the tube. The holes are drilled at one sixth of the length of the tube from both ends. Before mounting, the tube is circumferentially magnetized by passing 3 electrical current pulses $(1 \mathrm{~ms}$, $500 \mathrm{~A}$ ) through an insulated copper wire placed inside the Ni tube. The tube is surrounded by a glass tube containing the sample, which was thermostated at $25^{\circ} \mathrm{C}$. By applying a harmonic current through the transmitting coil, positioned in the middle of the tube, the tube is forced into torsional vibrations because of its magnetostrictive properties (Wiedemann effect). The motion of the tube is monitored in a second coil (inverse Wiedemann effect), positioned near the attachment of the upper pair of suspension wires. Besides the fundamental frequency, for which the length of the tube equals one half of a wavelength of the torsional waves, the tube shows many frequencies (overtones) for which resonance occurs. The overtones, for which the nodal planes coincide with the planes of the holes drilled in the tube, were used for measurements. The fundamental frequency was also used, although the requirement mentioned is not obeyed in this case. In practice it is found that the deviations of these measurements are relatively small. The sample holder could be closed to avoid evaporation.

Ellipsometer. This was used to obtain information on both the surface concentration and the thickness of the protein layer adsorbed at the air-saliva interface. It was equipped with a laser light source $(\lambda=632.8 \mathrm{~nm})$. The ellipsometric arrangement has been described elsewhere (De Feyter, Benjamins and Veer, 1978). A schematic view is given in Fig. 4. The linearly polarized monochromatic laser beam is con- 


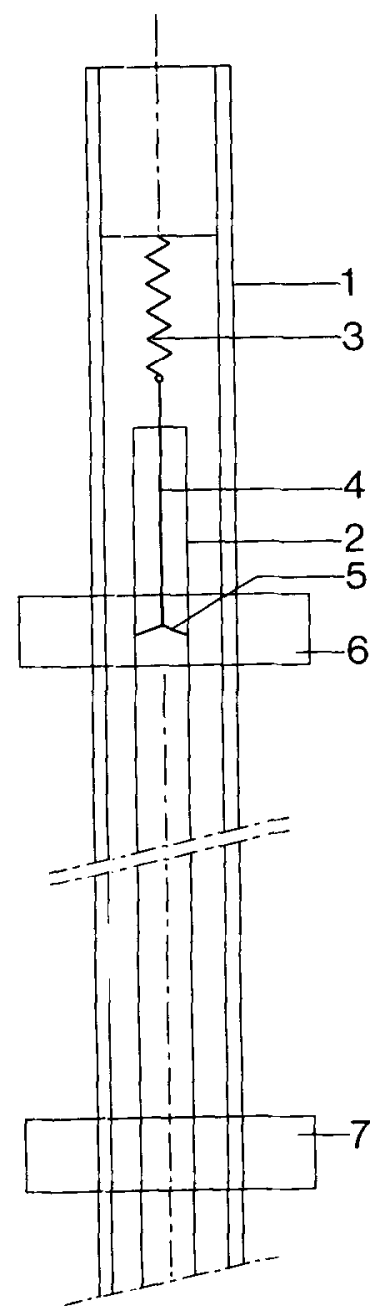

Fig. 3. Ni-tube resonator. 1-Glass tube; 2-Ni-tube; 3-spring; 4 -suspension wire; 5-knot; 6-measuring coil; 7-excitation coil.

verted into a circularly polarized beam by a quarter wave plate, the fast axis of which is positioned at an angle of $\pi / 4$ radians with the polarization plane of the laser beam. After passing through the polarizer, the beam is again linearly polarized, the polarization plane depending on the polarizer setting $(P)$. Subsequently it is converted into an elliptically polarized beam by the second quarter wave plate. In the arrangement used the second quarter wave plate was positioned at an angle of $\pi / 4$ radians with the plane of incidence. After reflection by the surface the reflected beam passes through an analyser and reaches a photomultiplier tube. Measurements involved adjustment of the polarizer in such a way that the elliptically polarized beam reaching the surface was reflected as a linearly polarized beam due to the optical properties of the reflecting system. If this was the case, adjustment of the analyser should result in an extinction of the reflected beam. The measured quantities were the extinction settings $(P)$ and $(A)$ of polarizer and analyser, respectively. In general there are more polarizer and analyser settings, falling in

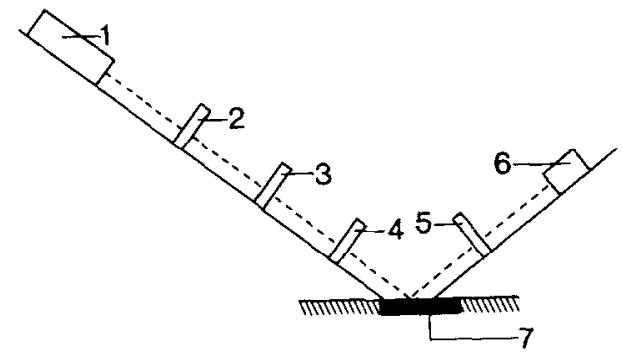

Fig. 4. Schematic drawing of the ellipsometer. 1-Laser; 2-quarter wave plate; 3-polarizer; 4-quarter wave plate; 5-analyser; 6-photomultiplier; 7-sample.

different zones, which produce extinction of the reflected beam. From the polarizer setting $(P)$ and the analyzer setting $(A)$ the ellipsometric parameters $(\psi)$ and $(\Delta)$ can be found. These parameters are defined as follows: the phase difference between the light components perpendicular and parallel to the plane of incidence is $\Delta$ and $\tan \psi$ is the ratio of the reflection coefficients of the perpendicular and parallel components.

In the arrangements described the following relationships hold

$$
\begin{aligned}
& \psi=A \\
& \Delta=(2 P+\pi / 2)
\end{aligned}
$$

From $\psi$ and $\Delta$ the thickness $h$ and the index of refraction $(n)$ of the layer can be calculated (Hansen, 1968).

\section{Procedures}

Steady-state measurements. To show unambiguously the presence of a surface layer, the following procedure was used. The sample holder was filled with the guard ring in the lower position. After $20 \mathrm{~min}$ a measurement was performed with a constant angular velocity of the cup of $1.39 \times 10^{-3} \mathrm{rad} \mathrm{s}^{-1}$. Subsequently the motor was stopped and the guard ring adjusted to its upper position. Again after a $20 \mathrm{~min}$ interval a measurement with the same angular velocity was performed. A 20 min wait time was applied as it takes some time for the surface layer to form and mature.

Measurements of the surface shear modulus. To study the rheological properties of the surface layer the sample holder of the torsional resonator was filled so that the sample just touched the bob (Fig. 5A). The harmonic oscillations of the resonator caused an outgoing cylindrical shear wave to propagate in the surface layer. This wave will be reflected at the sample holder into an ingoing cylindrical wave, which on its turn is reflected at the resonator, etc, leading to a standing wave pattern.

The bandwidth $\left(H B_{\mathrm{s}}\right)$ and the resonance frequency $\left(f_{0_{\mathrm{s}}}\right)$ of the resonance curve measured were compared with the corresponding reference data $\left(H B_{\mathrm{vac}}\right)$ and $\left(f_{0_{\text {vac }}}\right)$ of the unloaded (in vacuum) resonator. In practice the reference data were obtained for the resonator in air and a small correction must be applied. The changes of the bandwidths $\left(\triangle H B=H B_{\mathrm{s}}-H B_{\text {vac. }}\right)$ and of the resonance fre- 

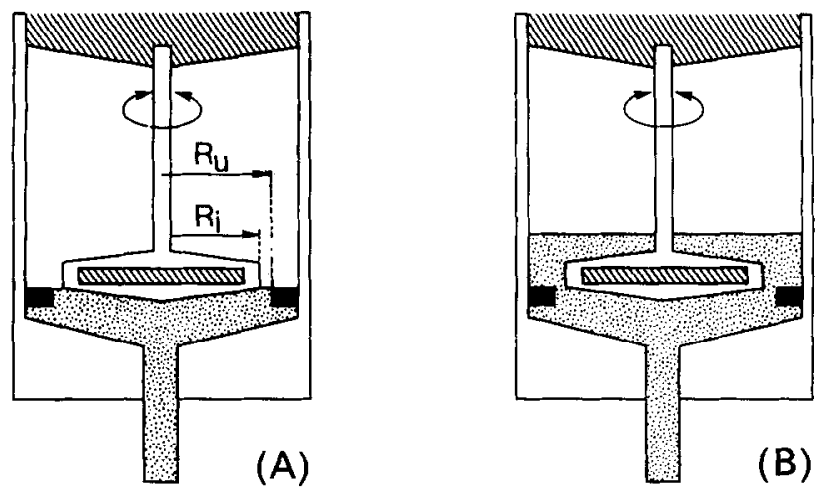

Fig. 5. Filling conditions of the torsional resonator. (A) Measurements of the surface laycr. (B) Measurements of the bulk liquid.

quencies $\left(\Delta f=f_{0_{\mathrm{s}}}-f_{0_{\mathrm{vac}}}\right)$ can be related to the restoring torque exerted by the sample on the bob. In general this torque will be out of phase with the torque exerted on the resonator by the exciting coil. For the in-phase and out-of-phase component it was found, respectively

$$
\begin{aligned}
M_{\text {in }} & =8 \pi^{2} I f_{0_{\text {vav }}} \cdot\left(f_{0_{\mathrm{s}}}-f_{0_{\text {vac }}}\right) \theta \\
M_{\text {out }} & =4 \pi^{2} I f_{0_{\text {vac. }}}\left(H B_{\mathrm{s}}-H B_{\text {vac. }}\right) \theta
\end{aligned}
$$

where $I$ equals the momentum of inertia of the bob. The restoring moment can be related to the mechanical properties of the surface layer. The underlying theory will be presented elsewhere, here only the formula will be given

$$
\begin{aligned}
M^{*} & =M_{\text {in }}+i M_{\text {out }}=2 \pi R_{i}^{3} \mu_{\mathrm{s}} k_{\mathrm{s}} \\
& \times \frac{\left[J_{2}\left(k_{\mathrm{s}} R_{i}\right) Y_{1}\left(k_{\mathrm{s}} R_{u}\right)-J_{1}\left(k_{\mathrm{s}} R_{\mathrm{u}}\right) Y_{2}\left(k_{\mathrm{s}} R_{i}\right)\right]}{\left[J_{1}\left(k_{\mathrm{s}} R_{i}\right) Y_{1}\left(k_{\mathrm{s}} R_{u}\right)-J_{1}\left(k_{\mathrm{s}} R_{u}\right) Y_{1}\left(k_{\mathrm{s}} R_{i}\right)\right]} \theta .
\end{aligned}
$$

In equation (4) the $k_{\mathrm{s}}$ denotes the (complex) wave number of the shear waves in the surface layer. $J_{1}(--)$ and $Y_{1}(--)$ are Bessel functions of the first order and of the first and second kind, respectively. $J_{2}(--)$ and $Y_{2}(-)$ are Bessel functions of the second order and of the first and second kind, respectively.

Combination of equations (3) and (4) leads to a relation between the complex quantities $M^{*}$, $\mu_{\mathrm{s}}=\mu_{\mathrm{s}}^{\prime}+i \mu_{\mathrm{s}}^{\prime \prime}$, the (complex) surface shear modulus and $k_{\mathrm{s}}$. Often $\mu_{\mathrm{s}}^{\prime}$ is called the surface shear modulus, while $\mu_{\mathrm{s}}^{\prime \prime}$ is connected to the surface shear viscosity $\left(\eta_{\mathrm{s}}\right)$ through

$$
\mu_{\mathrm{s}}^{t r}=\omega \eta_{\mathrm{s}} .
$$

To find $\mu_{\mathrm{s}}$ a second equation between the latter quantities is required. This second equation is formed by the so-called dispersion relation. The properties of the shear waves depend on the mechanical properties of both the surface layer and the bulk liquid underneath. To be more specific: the dispersion relation, i.e. the relation between the (complex) wave number $\left(\boldsymbol{k}_{\mathrm{s}}\right)$ and the angular frequency $(\omega)$ is determined by the surface density of the layer $\left(\rho_{s}^{\prime}\right)$ the density of the bulk liquid $\left(\rho_{1}\right)$ the complex surface shear modulus of the layer $\left(\mu_{\mathrm{s}}\right)$ and the complex viscosity $\left(\eta_{1}\right)$ of the bulk liquid. Waterman (1984) showed that for a homogeneous, plane-parallel, surface layer the dis- persion relation is in good approximation

$$
i \mu_{\mathrm{s}} k_{\mathrm{s}}^{2}=\rho_{1}\left(\omega^{2} / k_{\mathrm{l}_{0}}\right. \text {. }
$$

Equation (6) was given before by De Feyter (1978) in a somewhat different form for a Newtonian bulk liquid.

From equations (3), (4) and (6) it finally follows

$$
\begin{array}{r}
I f_{0_{\mathrm{vac}} .}\left(\Delta f+\frac{i \Delta H B}{2}\right)=-i 2 \pi R_{i}^{4} f_{0_{\mathrm{s}}}^{2} \rho_{1} k_{l_{0}}^{-1} \\
\times \frac{\left[J_{2}(\zeta) Y_{1}(\lambda \zeta)-J_{1}(\lambda \zeta) Y_{2}(\zeta)\right]}{\zeta\left[J_{1}(\zeta) Y_{1}(\lambda \zeta)-J_{1}(\lambda \zeta) Y_{1}(\zeta)\right]}
\end{array}
$$

where

$$
\zeta \equiv k_{\mathrm{s}} R_{i}
$$

and

$$
\lambda \equiv R_{u} / R_{i}
$$

Equation (7) shows how $\zeta=k_{\mathrm{s}} R_{i}$ can be deduced from the measured quantities $f_{0_{\mathrm{var}}}, f_{0_{\mathrm{s}}}, \Delta f, \Delta H B$, $\rho_{l}, k_{l_{0}}$ and the apparatus constants. $I, R_{i}$ and $R_{u}$. The complex wave number $\left(k_{s}\right)$ is buried in the arguments of the Bessel functions and numerical procedure is required to solve this equation. From $k_{\mathrm{s}}$ the surface shear modulus $\left(\mu_{\mathrm{s}}\right)$ is found from equation (6).

So far the influence of the bulk liquid on the measurements has not been dealt with.

Bulk liquid measurements. Because the wave number, $k_{\mathrm{l}_{0}}$, of the bulk liquid appears in the dispersion relation its shear modulus has to be measured as well. To this end the bob was completely immersed in the sample (Fig. SB). It can be demonstrated easily that the surface layer which adheres to the torsion rod has a negligible influence on the bulk liquid measurements. It was found that the bulk material was a low-viscosity liquid at the frequencies used. A detailed description of the measuring procedure in that case has been given before (Blom and Mellema, 1984). Measurements consist of the determination of the resonance frequencies $\left(f_{\mathrm{vac}}\right)$ and $\left(f_{1}\right)$ and the bandwidths $\left(H B_{\text {vac. }}\right)$ and $\left(H B_{1}\right)$ of the resonance curves measured with the empty and filled apparatus, respectively. From these measurements the real and imaginary part of the plane shear impedance 
$\left(Z_{\mathrm{pl.}}=R_{\mathrm{pl.}}+i X_{\mathrm{pl} .}\right)$ are found according to Blom and Mellema (1984).

$$
\begin{aligned}
\left(H B_{1}-H B_{\text {vac. }}\right) \equiv \Delta H B & =2 \mathrm{~K} R_{\mathrm{pl} .}+2 \mathrm{C} \eta^{\prime} \\
\left(f_{\mathrm{vac}}-f_{1}\right) \equiv \Delta f & =\mathrm{K} R_{\mathrm{pl} .}-\mathrm{C} \eta^{\prime \prime} .
\end{aligned}
$$

$\mathrm{K}$ and $\mathrm{C}$ are apparatus constants, $\eta^{\prime}$ and $\eta^{\prime \prime}$ are the real and imaginary part of the complex viscosity $\eta$. The dynamic viscosity is $\eta^{\prime}$, while $\eta^{\prime \prime}$ is connected to the shear modulus $G$

$$
\eta^{\prime \prime}=G / \omega
$$

Further we have

$$
\begin{gathered}
\eta^{\prime}=\frac{2 F_{\mathrm{pl} .} X_{\mathrm{pl} .}}{2 \pi f_{1} \rho_{1}} \\
\eta^{\prime \prime}=\frac{R_{\mathrm{pl} .}^{2}-X_{\mathrm{pl}}^{2}}{2 \pi f_{1} \rho_{1}}
\end{gathered}
$$

where $\rho_{1}$ is the density of the sample.

From (10), (11), (13) and (14) it is found

$$
Z_{\mathrm{pl} .}^{2}+\frac{2 \pi i f_{1} \rho_{1} \mathrm{~K}}{\mathrm{C}} Z_{\mathrm{pl} .}-\frac{2 h}{\mathrm{~K}}(\Delta H B+i \Delta f)=0 .
$$

The apparatus constants $\mathrm{K}$ and $\mathrm{C}$ are found by calibration of the instrument with Newtonian liquids with known viscosity and density.

The $k_{\mathrm{l}_{0}}$ then follows from:

$$
Z_{\mathrm{pl} .} \equiv \rho_{1} c_{\mathrm{l}_{0}}
$$

and

$$
k_{\mathrm{l}_{0}}=\omega / c_{\mathrm{l}_{0}}
$$

where $c_{10}$ is the complex velocity of the shear waves. The $\rho_{1}$ is measured with a PAAR DMA 40 digital density meter.

Correction for the bulk liquid. In performing surface layer measurements; part of the restoring torque exerted on the resonator will be due to the bulk liquid and a corresponding correction had to be applied. A first-order correction could be calculated from the results of the bulk liquid measurements. The existence of the surface layer, however, will affect the flow field in the bulk liquid and consequently the restoring torque. From the knowledge of the flow field (Waterman, 1984) it was found that this effect was negligible with respect to the first-order correction, which was applied throughout.

Ellipsometric measurements. Usually a measurement consists of two steps: determination of $\psi_{0}$ and $\Delta_{0}$ for the clean sclvent, which delivers the optical constants of the substrate, and determination of $\psi_{\mathrm{p}}$ and $\Delta_{p}$ of the solution in the presence of the surface layer. In our case a clean solvent was not available so that $\psi_{0}$ and $\Delta_{0}$ could not be measured directly. To overcome this difficulty a small amount of the bulk liquid was collected with a syringe. The refractive index of this material was determined with an Abbe refractometer, from which $\psi_{0}$ and $\Delta_{0}$ could be calculated. In a sample holder $20 \mathrm{ml}$ saliva was collected. Length, width and depth of this holder were 60,60 and $6 \mathrm{~mm}$, respectively. The extinction settings of both the polarizer and analyser were measured by their respective orientations at equal intensities on each side of the transmission minimum. From these settings $\psi_{p}$ and $\Delta_{p}$ were calculated from equations (1) and (2). In most cases $\psi_{\mathrm{p}}$ and $\Delta_{\mathrm{p}}$ were measured in two zones (McCrackin et al., 1963; Archer and Shank, 1967). Except in the first 10 min during which they changed very rapidly, $\psi_{\mathrm{p}}$ and $\Delta_{\mathrm{p}}$ could be measured to within $0.003^{\circ}$ and $0.02^{\circ}$, respectively. From the differences $\left(\delta \psi=\psi_{\mathrm{p}}-\psi_{0}\right)$ and ( $\delta \Delta=\Delta_{p}-\Delta_{0}$ ) the average values of the refractive index and the thickness of the adsorbed layer could be obtained, provided that the refractive index is a linear function of the solute concentration in the layer (De Feyter et al., 1978). The refractive index, $\mathrm{d} n / \mathrm{d} c$, for several oroteins are given elsewhere (Sober, 1968; De Feyter et al., 1978) and appear to be much the same, viz. $0.18 \mathrm{~cm}^{3} \mathrm{~g}^{-1}$. This value was used in this investigation.

\section{RESULTS}

\section{Steady-state measurements}

Figure 6 shows these: the arrows pointing downwards denote the moments at which the motor was switched on, the arrows pointing upwards when the motor was stopped. The lower curve shows the results with the guard ring in its lower position, the upper curve gives them with the guard ring in its upper position. For clarity the curves are shifted with respect to each other by an off-set voltage. From the lower curve it is seen that at the chosen angular velocity no measurable signal could be detected. With the guard ring in its upper position, however, a well-defined signal was found. The dip in the curve has no physical meaning. It shows that the surface layer is very fragile and that a small vibration, as was the case here, is sufficient to damage this layer a little. These results definitely show the presence of a surface layer. From this measurement a rough estimate of the apparent shear viscosity of the surface layer could be

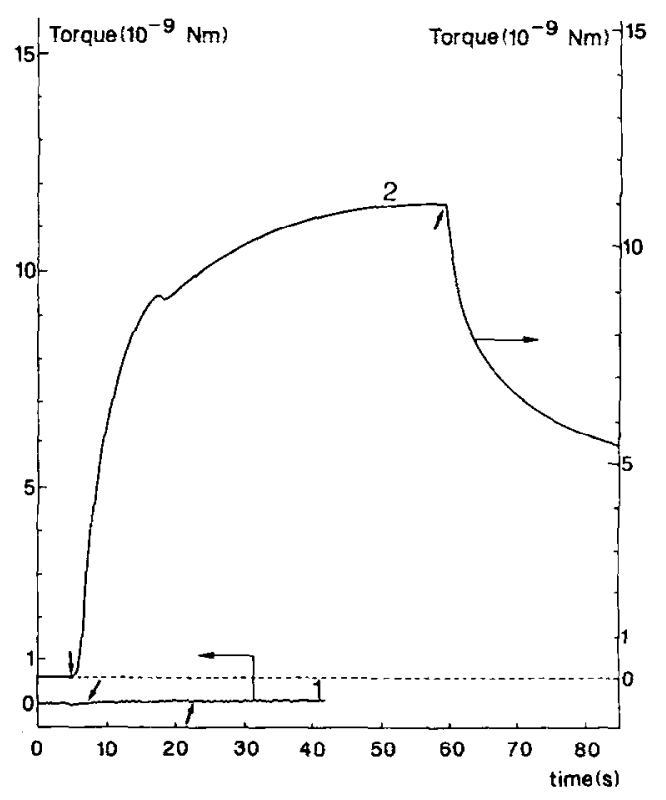

Fig. 6. The measured torque as a function of time obtained with the Contraves low shear 30 . Curve 1 : with guard ring in operation. Curve 2: with guard ring not in operation. 


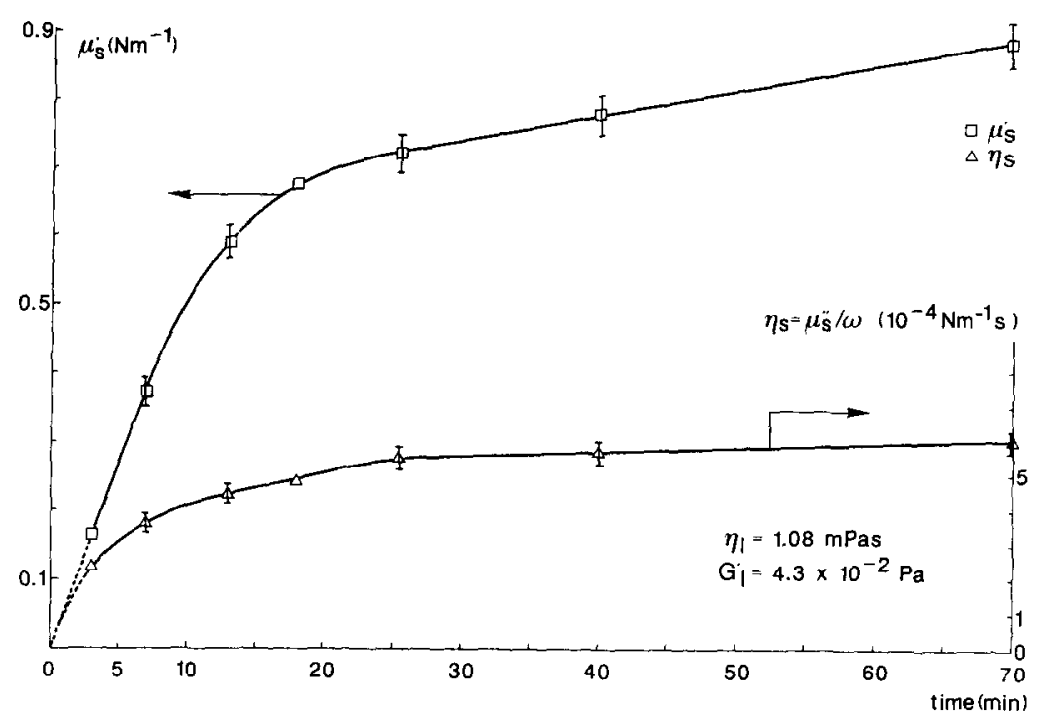

Fig. 7. Surface shear modulus $\left(\mu_{\mathrm{s}}^{\prime}\right)$ and surface shear viscosity $\left(\eta_{\mathrm{s}}\right)$ as a function of time.

made. The asymptotic value of the measured torque is in the order of $1 \times 10^{-8} \mathrm{Nm}$. This torque completely originates from the shearing of the surface layer. From this value the shear stress $\left(\tau_{R_{i}}\right)$ at the shaft of the bob and the shear rate $\left(\dot{\gamma}_{R_{i}}\right)$ at the shaft of the bob were calculated according to the following equations for wide gaps (Chmiel and Walitza, 1980)

$$
\begin{aligned}
& \tau_{R_{i}}=\frac{M}{2 \pi h R_{i}^{2}}\left\{\frac{1-\beta^{3}}{3(1-\beta)}\right\}^{1 / 2} \\
& \dot{\gamma}_{R_{i}}=2 \omega\left\{\frac{1-\beta^{3}}{3(1-\beta)^{3}}\right\}^{1 / 2}
\end{aligned}
$$

where $\beta=\left(R_{i} / R_{u}\right)^{2}$ and $h$ is the thickness of the surface layer. From equations (18) and (19) and $h=100 \mathrm{~nm}$ the apparent viscosity of the surface layer in this non-linear experiment was estimated from $\eta=\tau_{R_{i}} / \gamma_{R_{i}}$ to be about $10^{6} \mathrm{~Pa}$ s. Here $R_{i}$ equals the radius of the shaft of the bob and $R_{\mathrm{s}}$ the outer radius of the surface layer.

\section{Surface shear modulus and surface shear viscosity}

In Fig. 7 the surface shear modulus $\left(\mu_{s}^{\prime}\right)$ and the surface shear viscosity $\left(\eta_{s}\right)$ of submandibular saliva are shown as a function of the surface age. This figure indicates a rapid growth during the first $10-15 \mathrm{~min}$ for both $\mu_{\mathrm{s}}^{\prime}$ and $\eta_{\mathrm{s}}$ after which these quantities gradually increase. As is common in these measurements the zero value of the time axis is somewhat uncertain.

It was not possible to perform measurements in the regime of linear viscoelasticity. [Roughly speaking, in this regime, doubling the input signal results in a doubling of the output signal, letting the complex (surface) shear modulus unimpaired]. In Fig. 8 the values of $\mu_{\mathrm{s}}^{\prime}$ and $\eta_{\mathrm{s}}$ are shown as a function of the shear deformation for a surface layer with an age of $80 \mathrm{~min}$. The smallest shear deformation applied, viz. $4 \times 10^{-5}$, corresponds to an angular amplitude of the bob of $10^{-5}$ rad. Decreasing this amplitude resulted in signal to noise ratios too bad to produce accurate data.

\section{(Complex) viscosity of the bulk liquid}

In Fig. 9 the real and imaginary parts of the complex viscosity $\left(\eta=\eta^{\prime} \cdots\right.$ i $\left.\eta^{\prime \prime}\right)$ are given. The real part $\left(\eta^{\prime}\right)$ of the complex viscosity decreases from $1.1 \mathrm{mPa} \mathrm{s}$ at $70 \mathrm{~Hz}$ to approx. $0.95 \mathrm{mPa} \mathrm{s}$ at high frequencies. The path of $\eta^{\prime \prime}$ is similar. For $\eta^{\prime \prime}$ a bell-shaped curve was expected with a maximum at a certain transition frequency and decreasing to small values on both sides of the maximum. This behaviour is to be expected because the measurements were linear ones and the mentioned behaviour is deduced from a general theory of linear viscoelastic bchaviour (Flügge, 1975). For comparison, the viscosity of water at the same temperature equals $0.89 \mathrm{mPa}$ s.

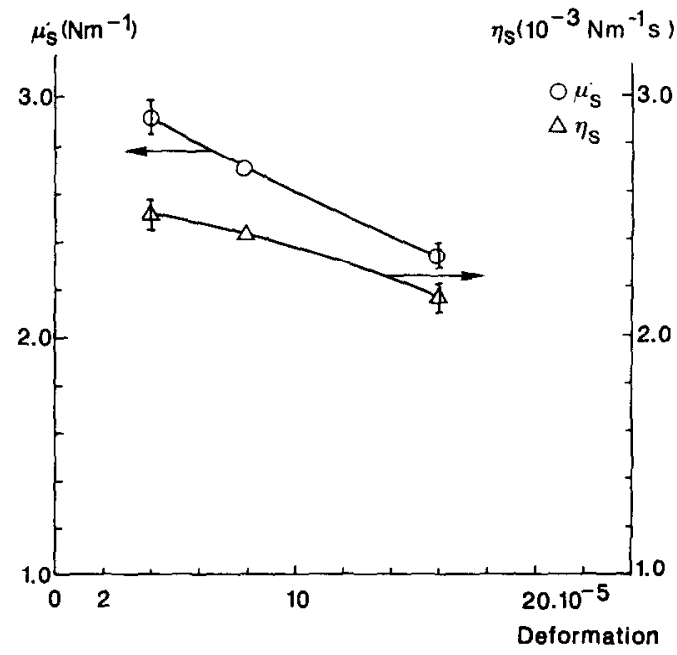

Fig. 8. The surface shear viscosity $\left(\mu_{\mathrm{s}}^{\prime}\right)$ and the surface shear viscosity $\left(\eta_{\mathrm{s}}\right)$ as a function of deformation. 


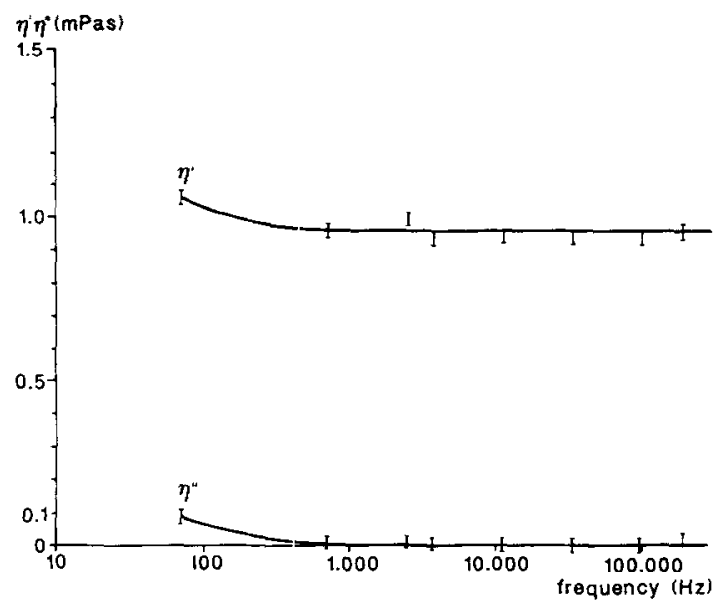

Fig. 9. The real part $\left(\eta^{\prime}\right)$ and the imaginary part $\left(\eta^{\prime \prime}\right)$ of the complex viscosity $\left(\eta=\eta^{\prime}-\eta^{\prime \prime}\right)$ of the bulk liquid versus frequency.

\section{Ellipsometric measurements}

In Fig. 10 the surface concentration $(\Gamma)$ is plotted as a function of the age of the surface layer. The surface concentration was high as compared with the generally found surface concentration of aqueous protein solutions. In the same figure the thickness $(h)$ of the layer is plotted. Here, the values were also much higher than generally found for aqueous protein solutions. In both curves the measuring points at $t=10 \mathrm{~min}$ deviate strongly from a smooth curve through the measuring points. In the curves these points were omitted. Their paths strongly resemble the $\mu_{s}^{\prime}-t$ curves. From the refractive indices of the bulk liquid and water measured with the same refractometer the protein concentration was found to be $(4 \pm 1) \mathrm{mg} \mathrm{m}^{-1}$.

At the same time the surface tension was measured with a Wilhelmy plate. After an initial rapid decrease during the first minutes, the value of the surface tension levelled off until a value of $46 \mathrm{mNm}^{-1}$ and finally even increased. The reason for this aberration appeared to be the freezing-in of the Wilhelmy plate in the surface layer together with the drying out of the bulk liquid below the layer. After a small vertical movement of the Wilhelmy plate the original value $\left(49 \mathrm{mNm}^{-1}\right)$ reduced to $40 \mathrm{mNm}^{-1}$.

\section{DISCUSSION}

The existence of an adsorbed layer at the liquid-air interface of aqueous protein solutions has been known for about half a century, Joly (1939) being one of the first investigators to study its mechanical properties. To the best of our knowledge, however, the presence of such a layer in saliva, and especially its pronounced viscoelastic properties, have not been mentioned in the literature. Measurements of the surface shear modulus of the adsorbed layer at the air interface of aqueous solutions of natural proteins, viz. sodium caseinate and bovine serum albumin at a frequency of about $3 \times 10^{-2} \mathrm{~Hz}$, have been reported (De Feyter and Benjamins, 1979). The values of $\mu_{\mathrm{s}}^{\prime}$ and $\mu_{s}^{\prime \prime}$ of these materials are about $10^{-3} \mathrm{Nm}^{-1}$; the thicknesses $(d)$ of the layers are about $4 \mathrm{~nm}$. For the bulk shear moduli $\left(G_{\mathrm{s}}=\mu_{\mathrm{s}}^{\prime} / d\right)$ of these layers values of about $10^{5} \mathrm{~Pa}$ were found. Combining the results of our surface shear measurements and our ellipsometric measurements, the bulk shear modulus of the salivary surface layer was found to be about $10^{7} \mathrm{~Pa}$ and the bulk viscosity of the layer about $10^{4} \mathrm{~Pa}$ s. The big difference between the surface shear moduli of the natural proteins mentioned and that of saliva therefore is largely due to differences in the thicknesses of these layers. Surface dilatational moduli of the same magnitude as we found for the surface shear moduli are reported for fairly insoluble monolayers after collapse (Veer and van den Tempel, 1973). The collapse of the monolayer, as formed in a Langmuir trough, is caused by decreasing its area about 25 per cent. After collapse, gel-like or mesomorphous particles resembling liquid crystals are present in the surface. The elasticity of surfaces containing such multilayers is much higher than the

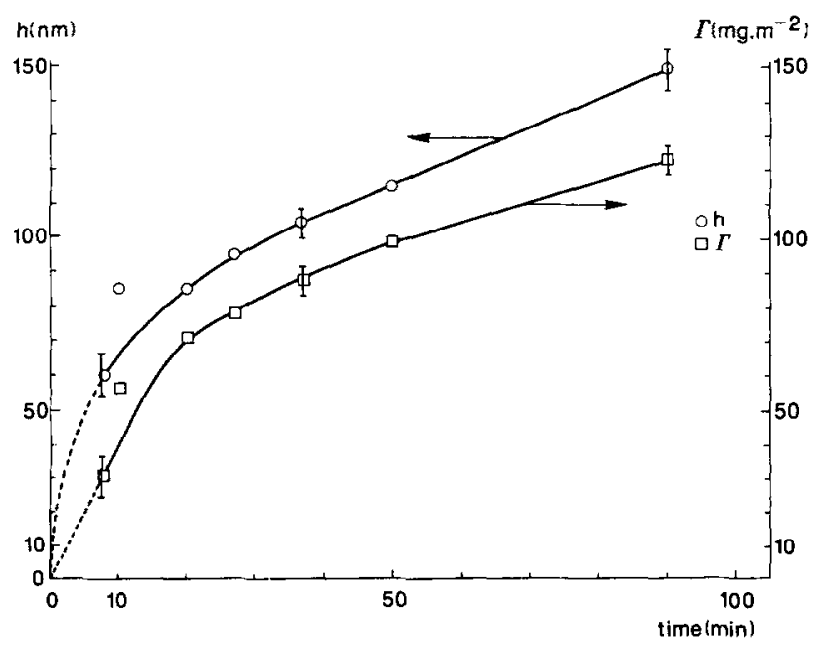

Fig. 10. Thickness $(h)$ and surface concentration $(\Gamma)$ of the layer as a function of time. 
elasticity of monolayers, and also the viscosity is very high (Veer and van den Tempel, 1973). The large thicknesses of the layers found in our experiments suggest the existence of a multilayer.

The finding of a highly elastic surface layer at the saliva-air interface questions the interpretation of saliva measurements made with various theological instruments. For example, during measurements with a Weissenberg Rheogoniometer (Davis, 1971; Briedis, Moutrie and Balmer, 1980; Schwarz, 1987), the torque detected is partly due to the surface layer at the saliva-air interface. It is recommended that in future work the influence of a surface layer on saliva is considered in any interpretation.

Comparison of the viscosity of saliva above $70 \mathrm{~Hz}$ (without the influence of the surface layer) with values found in the literature is hampered in three respects: firstly, as mentioned, the influence of the surface layer on previous values is unknown; secondly it appears that the linearity of the harmonic measurements was not always checked; and thirdly the dynamic viscosity was only reported at much lower frequencies (typically $10^{-2}-10 \mathrm{~Hz}$ ). However, the theoretically expected (Flügge, 1975) trend, namely that with increasing frequency $\eta^{\prime}$ decreases and $G$ increases to limit values, is consistent with the previously observed behaviour at low frequencies and with our measurements above $70 \mathrm{~Hz}$. Preliminary measurements in our laboratory between 0.03 and $3 \mathrm{~Hz}$, excluding surface layer influence and checking the linearity, confirm this trend.

Acknowledgements-The ellipsometer used in this investigation was made available to us by the courtesy of $\mathrm{Dr}$ J. A. de Feyter and Mr J. Benjamins (Unilever Research, Vlaardingen, The Netherlands). We thank our colleague Professor Dr L. van de Klundert for providing us with the computer program to calculate the complex wave number $\left(k_{\mathrm{s}}\right)$ from equation (7).

\section{REFERENCES}

Archer R. J. and Shank C. V. (1967) Filipsometry with non-ideal compensators. J. Opt. Soc. Am. 57, 191-194.

Balmer R. T. S. and Hirsch S. R. (1978) The non-Newtonian behaviour of human saliva. AIChE Symposium Series on Biorheology No. 182 74, 125-129.

Blom C, and Mellema J. (1984) Torsion pendula with electromagnetic drive and detection system for measuring the complex shear modulus of liquids in the frequency range $80-2500 \mathrm{~Hz}$. Rheol. Acta $23,98-105$
Briedis D., Moutrie M. F. and Balmer R. T. (1980) A study of the shear viscosity of human whole saliva. Rheol, Acta $19,365-374$.

Chmiel H. and Walitza E. (1980) On the Rheology of Blood and Synovial Fluids, p. 95. Wiley, New York.

Davis S. S., (1971) The rheological properties of saliva. Rheol. Acta 10, 28-35.

De Feyter J. A. (1978) The propagation of surface shear waves. I. Theory. J. Colloid Interface Sci. 69, 375-383.

De Feyter J. A. and Benjamins J. (1979) The propagation of surface shear waves. II. Experiments. J. Colloid Interface Sci. 70, 375-382

De Feyter J. A., Benjamins J. and Veer F. A. (1978) Ellipsometry as a tool to study the adsorption behaviour of synthetic- and biopolymers at the air-water interface. Biopolymers 17, 1759-1772.

Flügge W. (1975) Viscoelasticity, Springer, Berlin.

Hansen W. N. (1968) Electric fields produced by the propagation of plane coherent electromagnetic radiation in stratified medium. J. Opt. Soc. Am. 5, 380-390

Joly M. (1939) Viscosity of surface layers of proteins. $J$. chim. Phys. 36, 285-295.

Lashley K. S. (1916) Reflex secretion of the human parotid gland. J. exp. Psychol. 1, 461-463.

Mandel I. D. and Wotman S. (1976) The salivary secretions in health and disease. Oral Sci. Rev, 8, 25-47.

McCrackin F. L., Passaglia E., Stromberg R. R. and Steinberg L. (1963) Measurement of the thickness and refractive index of very thin films and the optical properties of surfaces by ellipsometry. J. Res. natn. Bur. Stand. Section A 67, 363-377

Oosterbroek M., Waterman H. A., Wiseall S. S., Altena E. G., Mellema J. and Kip G. A. M. (1980) Automatic apparatus, based upon a nickel-tube resonator, for measuring the complex shear modulus of liquids in the $\mathrm{kHz}$ range. Rheol. Acta 19, 497-506.

Schneyer L. H. (1955) Method for the collection of separate submaxillary and sublingual salivas in man. $J$. dent. Res 34, 257-261.

Schwarz W. H. (1987) The rheology of saliva. J. dent. Res. (special issue) 66, 660-666.

Sober H. A. (Ed.) (1968) Handbook of Biochemistry. The Chemical Rubber Co., Cleveland.

Veer F. A. and Tempel M. van den (1973) Surface tension relaxation in a surface containing surfactant particles. $J$. Colloid Interface Sci. 42, 418-426.

Waterman H. A. (1984) Measurement of the dynamic shear modulus of surface layers. I. Theory. J. Colloid Interface Sci. 101, 377-383.

Waterman H. A., Oosterbroek M., Beukema G. J. and Altena E. G. (1979) On the use of a nickel-tube resonator for measuring the complex shear modulus of liquids in the kHz-range. Rheol. Acta 18, 585-592.

Waterman H. A., 's-Gravenmade E. J. and Wallen-Mijniieff P. F. van der (1985) Time dependent rheological behaviour of human saliva. $J$. dent. Res. 64, 716 . 